\title{
Spontaneous Vesiculation and pH-Induced Disassembly of a Lysosomotropic Detergent: Impacts on Lysosomotropism and Lysosomal Delivery
}

Ana Maria Villamil Giraldo, Timmy Fyrner, Stefan Wennmalm, Atul N. Parikh, Karin Öllinger and Thomas Ederth

\section{Journal Article}

\section{Tweet}

N.B.: When citing this work, cite the original article.

Original Publication:

Ana Maria Villamil Giraldo, Timmy Fyrner, Stefan Wennmalm, Atul N. Parikh, Karin Öllinger and Thomas Ederth, Spontaneous Vesiculation and $\mathrm{pH}$-Induced Disassembly of a Lysosomotropic Detergent: Impacts on Lysosomotropism and Lysosomal Delivery, LANGMUIR, 2016. 32(50), pp.13566-13575.

http://dx.doi.org/10.1021/acs.langmuir.6b03458

Copyright: American Chemical Society http://pubs.acs.org/

Postprint available at: Linköping University Electronic Press

http://urn.kb.se/resolve?urn=urn:nbn:se:liu:diva-134081 


\section{Spontaneous vesiculation and $\mathrm{pH}$-induced disassembly of a lysosomotropic detergent: impacts on lysosomotropism and lysosomal delivery}

Ana M. Villamil Giraldo ${ }^{\mathrm{a}}$, Timmy Fyrner ${ }^{\mathrm{b}}$, Stefan Wennmalmº, Atul N. Parikh ${ }^{\mathrm{d}}$, Karin Öllinger ${ }^{\mathrm{a}, *}$, Thomas Ederth ${ }^{\mathrm{e}, *}$

${ }^{a}$ Experimental Pathology, Department of Clinical and Experimental Medicine, Linköping University, SE-581 85 Linköping, Sweden.

${ }^{b}$ Division of Chemistry, Department of Physics, Chemistry and Biology, Linköping University, Linköping SE-581 83, Sweden.

c Scilifelab, Royal Institute of Technology, Department of Applied Physics, Experimental Biomolecular Physics, 17165 Solna, Sweden.

${ }^{d}$ Departments of Biomedical Engineering and Materials Science \& Engineering, University of California, Davis, California 95616 USA.

${ }^{e}$ Division of Molecular Physics, Department of Physics, Chemistry and Biology, Linköping University, Linköping SE-581 83, Sweden.

* Corresponding authors: karin.ollinger@liu.se, ted@ifm.liu.se

Published as: A. M. Villamil Giraldo, T. Fyrner, S. Wennmalm, A. N. Parikh, K. Öllinger, T. Ederth, "Spontaneous vesiculation and $\mathrm{pH}$-induced disassembly of a lysosomotropic detergent: impacts on lysosomotropism and lysosomal delivery." Langmuir, 32(50), 1356613575 (2016). DOI: 10.1021/acs.langmuir.6b03458. 


\begin{abstract}
Lysosomotropic detergents (LDs) selectively rupture lysosomal membranes through mechanisms that have yet to be characterized. A consensus view, currently, holds that LDs, which are weakly basic, diffuse across cellular membranes as monomers in an uncharged state, and via protonation in the acidic lysosomal compartment, they become trapped and accumulate, and subsequently solubilize the membrane and induce lysosomal membrane permeabilization. Here, we demonstrate that the lysosomotropic detergent $O$-methyl-serine dodecylamide hydrochloride (MSDH) spontaneously assembles into vesicles at, and above, cytosolic $\mathrm{pH}$, and that the vesicles disassemble as $\mathrm{pH}$ reaches 6.4 or lower. The aggregation commences at concentrations below the range of those used in cell studies. Assembly and disassembly of the vesicles was studied via dynamic light scattering, zeta potential measurements, cryo-TEM and fluorescence correlation spectroscopy, and was found to be reversible via control of the $\mathrm{pH}$. Aggregation of MSDH into closed vesicles under cytosolic conditions is at variance with the commonly held view of LD behaviour, and we propose that endocytotic pathways should be considered as possible routes of LD entry into lysosomes. We further demonstrate that MSDH vesicles can be loaded with fluorophores via a solution transition from low to high $\mathrm{pH}$, for subsequent release when the $\mathrm{pH}$ is lowered again. The ability to encapsulate molecular cargo into MSDH vesicles together with its ability to disaggregate at low $\mathrm{pH}$ and to permeabilize the lysosomal membrane presents an intriguing possibility to use MSDH as a delivery system.
\end{abstract}




\section{Introduction}

Lysosomes are subcellular organelles with an acidic interior ( $\mathrm{pH}$ 3.5-5), surrounded by a single bilayer membrane, and contain hydrolytic enzymes that break down macromolecules and intracellular waste. The acidic interior makes lysosomes susceptible to accumulation of weakly basic substances, such as amines, which diffuse across the lysosomal membrane in their uncharged state, but as they reach the acidic interior and become protonated, transmembrane diffusion of the now charged molecule through the lipid bilayer back to the cytosol is severely hindered. Such substances are referred to as lysosomotropic agents ${ }^{1}$, and via this mechanism, trapped substances may accumulate to concentrations hundredfold that of the cytosolic concentration. ${ }^{2}$ Firestone and co-workers ${ }^{3,4}$ synthesized a series of basic detergents, such as alkyl amines and alkyl imidazoles, which are largely un-ionized at the pH of the cytosol, and selectively accumulate in lysosomes. However, in contrast to other lysosomotropic agents, it was suggested that these lysosomotropic detergents (LDs) acquire detergent properties within the lysosomes, while lacking surface activity in the cytosol. It was further hypothesized that at sufficiently high concentrations they destabilize the lysosomal membrane, eventually leading to leakage of the lysosomal content, and ultimately to cell death. ${ }^{5}$ The cytotoxicity of the LD types originally devised by Firestone ${ }^{3}$, is well documented, including $N$-dodecylimidazole ${ }^{5,6,7,8}, N$-dodecylmorpholine ${ }^{6}$, and fluorinated $N$ dodecylamines $^{9}$. Also serine alkylamides ${ }^{4,10}$ as well as structurally very different compounds such as siramesine ${ }^{11}$ are cytotoxic LDs. Additionally, LDs have been demonstrated to exert

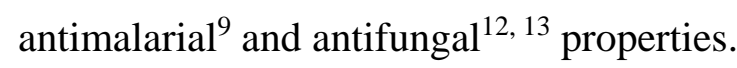

It was initially suggested that LDs enter the lysosomes along the same route, and under the same conditions, as other lysosomotropic agents, ${ }^{3}$ but it appears that this proposition has never been tested. Experiments by Miller et al. suggested that LDs indeed kill cells by disrupting lysosomes from within ${ }^{5}$, and in the earlier work by Firestone ${ }^{3}$, a chain-length dependence on the toxicity of different alkyl amines was evident, indicating a physical rather than a chemical origin of the toxicity, and also supporting detergency as the mode of action. Cells lacking lysosomes, such as erythrocytes ${ }^{3}$, were found to be insensitive to LDs, further indicating that lysosomes are required for LD toxicity. However, in an experiment designed to test the effects of LDs on the integrity of lysosomal membranes, Forster et al. did not see any evidence of destabilizing effects by LDs on either isolated lysosomes or on lysosomes in fibroblast cells, ${ }^{6}$ and suggested that the cytotoxic effect may be mediated by a non-lysosomal mechanism. In a subsequent study, the results were mixed, and it was proposed that this was 
due to differences in susceptibility to lysosomal membrane permeabilization, i.e. that some cells have their lysosomal membranes permeabilized and die, whereas resistant cells do not, and remain viable. However, no further explanation or reason for this difference was provided. ${ }^{14}$ Similarly, Cabantchik and co-workers failed to observe any detergent-like action of LDs on the acidic compartments of malarial parasites ${ }^{9}$. Siramesine destabilizes the lysosomal membrane ${ }^{11}$, but is structurally different from alkyl LDs, and while it partitions into bilayers, it also binds to acidic phospholipid headgroups with high affinity, and sequestering of acidic lipids has been suggested as an alternative mechanism by which it contributes to cell toxicity. ${ }^{15}$

The sigmoidal concentration dependence of toxicity on LD concentration ${ }^{5,7,12,16}$, indicates cooperative behaviour ${ }^{5,12}$ and has been linked to aggregation, or the critical micelle concentration (CMC), of the protonated LD. ${ }^{3}$ In the prevailing picture, LDs are assumed not to harm plasma membranes of cells, but diffuse freely across these, because as free bases they are lipophilic and not surface active, but become powerful detergents upon protonation, ${ }^{13}$ or phrased differently, "the largely un-ionized amine will be simply an oily substance without surface-active properties, but upon ionization within the lysosome it will become a detergent”3. This is questionable on physicochemical grounds, since LDs will still have more or less polar headgroups under neutral conditions, and charging of headgroups usually counteracts aggregation due to headgroup Coulomb repulsion. This is supported and exemplified by the $\mathrm{pH}$ dependence on the CMC of sphingosine, a natural sphingolipid with lysosomotropic detergent ability, for which the CMC changes from $0.7 \mu \mathrm{M}$ in the deprotonated state, to $1.7 \mu \mathrm{M}$ in the protonated state, with a shift at $\mathrm{pH} 6.6$, indicating a higher propensity for aggregation in the cytosol than in lysosomes. ${ }^{17}$

The mechanism(s) by which lysosomotropic detergents destabilize lysosomes remains unknown, and the currently held view of how LDs enter cells and rupture lysosomal membranes has not been verified. The experimental evidence in this matter raises several important questions, which have remained unanswered. These concern the aggregation state of LDs in the cytosol and in the lysosomes, the mode of entry through the plasma and lysosomal membranes, the effects of $\mathrm{pH}$ and intracellular environments on the aggregation or dispersal of LDs, the exent to which LDs partition into, or solubilize, the inner leaflets of lysosomal membranes, and what qualitative differences there are in the behaviour and effects of different LDs. These issues are central to understanding lysosomotropism, and must be adressed in order to understand the mechanism(s) of lysomotropic detergent action, and their 
contributions to cytotoxicity, at the molecular level. While LDs have been extensively used for controlled release of lysosomal content, ${ }^{10,18,19,20}$ interest in lysosomotropic agents is not limited to destabilization of lysosomes. Lysosomotropic agents are also used for fluorescenceguided surgery ${ }^{21}$, as lysosomal markers in fluorescence microscopy (acridine orange, LysoTracker ${ }^{\circledR}$ ), or used as lysosomal transporters via covalent conjugation ${ }^{22}$.

The synthetic LD O-methyl-serine dodecylamide hydrochloride (MSDH) (see Figure 1) is one in a series of LDs initially designed as anticancer drugs ${ }^{3,16}$, and has also been tested for its ability to inhibit DNA synthesis. ${ }^{4}$ It selectively ruptures lysosomes, but is otherwise biochemically inactive, facilitating study of the specific mechanisms of lysosome-dependent cell death. Cell studies unequivocally demonstrate that MSDH selectively causes lysosomal membrane permeabilization. ${ }^{10,19}$

The specificity of MSDH towards lysosomes has been explained by its specific protonation, and ensuing accumulation, inside these organelles where $\mathrm{pH}$ is 5 or lower. ${ }^{4}$ However, the actual mechanism by which MSDH, or indeed any other lysosomotropic detergent, enter the lysosomes, or how they permeabilize the membrane to release lysosomal content, have never been investigated. With the prospects of gaining insight into the events taking place in whole cells, we examined the aggregation properties of MSDH under various $\mathrm{pH}$ conditions, using a combination of cryo-TEM, dynamic light scattering, zeta potential measurements, and also assessed the loading capability of MSDH vesicles using fluorescent quenching assays and fluorescence correlation spectroscopy. We discuss the aggregation and the lysosomotropic properties of MSDH in detail, and also comment on the possible use of MSDH for lysosomal drug delivery.

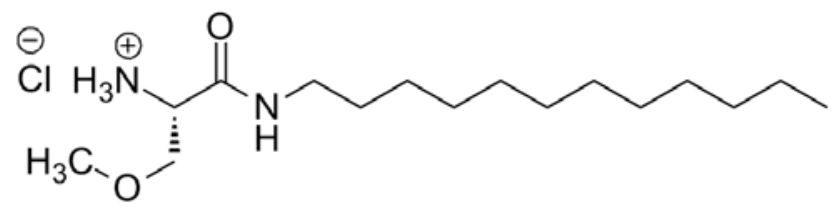

Figure 1. O-methyl-serine dodecylamide hydrochloride (MSDH).

\section{Materials and Methods}

MSDH synthesis MSDH was synthesized according to a published protocol ${ }^{4}$, purified using reverse phase chromatography (Merck LiChroprep $\left.{ }^{\circledR}(\mathrm{RP}-18)\right)\left(95: 5 \rightarrow 50: 50 \mathrm{H}_{2} \mathrm{O} / \mathrm{MeOH}+\right.$ 
$0.1 \% \mathrm{HCl}$ (aq.)) and lyophilized from $\mathrm{H}_{2} \mathrm{O}$ to give an off-white solid. ${ }^{1} \mathrm{H}$ - and ${ }^{13} \mathrm{C}$ NMRspectra were in accordance with those reported earlier ${ }^{4}$. Optical rotation measurements were recorded at $20{ }^{\circ} \mathrm{C}$ with a Perkin-Elmer 141 polarimeter; $[\alpha]_{\mathrm{D}}-9.1\left(c 1, \mathrm{H}_{2} \mathrm{O}\right)$. The original synthesis protocol ${ }^{4}$ uses reaction conditions that can give rise to scrambling of the chiral center, but an enantiomeric excess (ee) of 95.2\% was determined using resolution gas chromatography after chemical derivatization with (S)-(-)- $N$-(trifluoroacetyl)pyrrolidine-2carbonyl chloride. Electrospray ionisation mass spectrometry (ESI-MS) (Water Synapt HDMS with electrospray interface, recorded at Medivir AB, Huddinge, Sweden): [M] ${ }^{+}$ calculated for $\mathrm{C}_{16} \mathrm{H}_{34} \mathrm{~N}_{2} \mathrm{O}_{2}$, 286.2621; found 286.2613.

Dynamic light scattering (DLS) DLS measurements were performed using an ALV-CGS5022F Goniometer system (ALV GmbH, Langen) with a $22 \mathrm{~mW}$ HeNe laser illuminating the sample in a cylindrical cuvette immersed in refractive-index-matching toluene. The toluene temperature is controlled to $22 \pm 0.02{ }^{\circ} \mathrm{C}$ using a circulating water bath and samples were equilibrated in the circulating water for $\geq 10 \mathrm{~min}$ before measurements. Scattered light is detected at $90^{\circ}$ via a near single-mode optical fiber, split to two avalanche photodiodes (Perkin-Elmer) for operation in pseudo-cross-correlation mode. A dual multiple-tau digital correlator with 328 channels (ALV-6010-160) yields the time-correlation function of the scattered intensity. Correlation time distributions were calculated using a constrained regularization method supplied with the correlator software and the correlation time distribution recalculated to intensity size distributions via the Stokes-Einstein relationship. Zaveraged particle sizes were obtained via second order cumulants analysis, provided with the correlator software, ignoring correlation data beyond $10 \%$ of the maximum value.

Zeta potential The zeta potentials were measured using a Zetasizer Nano ZS90 (Malvern Instruments Ltd, Worcestershire, UK). Measurements were performed after titrating the suspensions of MSDH vesicles to the different $\mathrm{pH}$ values using disposable folded capillary cells (DTS 1070) previously rinsed with ethanol and MilliQ water ( $>18.2 \mathrm{M} \Omega \mathrm{cm}$, Millipore).

Preparation of vesicles for DLS and zeta potential measurements MSDH was resuspended in MilliQ water to a final concentration of $60 \mathrm{mM}$ and extruded 21 times through $100 \mathrm{~nm}$ pore size polycarbonate membrane filters (Nucleopore). This suspension was diluted 100 times in $10 \mathrm{mM}$ HEPES $10 \mathrm{mM} \mathrm{KCl}$ at the different tested $\mathrm{pH}$ values. $\mathrm{pH}$ measurements (using an MT SevenEasy $\mathrm{pH}$ meter) were routinely preceded by a two-point calibration at $\mathrm{pH} 4$ and $\mathrm{pH} 7$, 
and sometimes three-point calibrations with an additional point at $\mathrm{pH} 10$. The $\mathrm{pH}$ measurement error is estimated to be $<0.03 \mathrm{pH}$ units.

Loading and release of carboxyfluorescein in MSDH vesicles $40 \mathrm{mg}$ MSDH was resuspended in $200 \mu \mathrm{l}$ of $10 \mathrm{mM}$ HEPES pH 5.3, $10 \mathrm{mM} \mathrm{KCl}$ and mixed with $1.6 \mathrm{ml}$ of a highly concentrated solution of carboxyfluorescein (CXF): 106 mM CXF, 50 mM HEPES pH 8.6. In this final solution MSDH and CXF concentrations were $69 \mathrm{mM}$ and $94 \mathrm{mM}$, respectively, and pH was 7.5. Non-encapsulated CXF was removed by size-exclusion chromatography using a pre-packed Sephadex column (PD MiniTrap G10, GE Healthcare), equilibrated with a $10 \mathrm{mM}$ $\mathrm{KCl}+10 \mathrm{mM}$ HEPES buffer at $\mathrm{pH}$ 7.2. $0.5 \mathrm{ml}$ of sample was loaded, and eluted in $1 \mathrm{ml}$ of buffer. Fluorescence ( $\lambda_{\text {exc }} 494 \mathrm{~nm}, \lambda_{\text {em }} 520 \mathrm{~nm}$ ) was measured in a Fluoromax4 spectrophotometer at room temperature before and after the addition of Triton X-100 (0.1 \% final concentration), in the same buffer.

Loading and release of Rhodamine 6G in MSDH vesicles $40 \mathrm{mg}$ MSDH was resuspended in 1 $\mathrm{ml}$ of $10 \mathrm{mM}$ HEPES pH 5.3, $10 \mathrm{mM} \mathrm{KCl} .150 \mu \mathrm{l}$ of this suspension was mixed with $350 \mu \mathrm{l}$ of Rhodamine 6G $1 \mu \mathrm{M}$ in $50 \mathrm{mM}$ HEPES pH $8.650 \mathrm{mM} \mathrm{KCl}$. In this final solution MSDH and Rhodamine 6G concentrations were $37 \mathrm{mM}$ and $0.7 \mu \mathrm{M}$, respectively, and $\mathrm{pH}$ was 7.5. Non-encapsulated Rhodamine 6G was removed using a pre-packed Sephadex column (PD MiniTrap G10, GE Healthcare). The sample was diluted to $6 \mathrm{mM}$ MSDH concentration in a $10 \mathrm{mM} \mathrm{KCl,} 10 \mathrm{mM}$ HEPES buffer at $\mathrm{pH} 7.2$ or at $\mathrm{pH} 4$.

Fluorescence correlation spectroscopy FCS measurements were performed on a Zeiss 780 confocal microscope (Zeiss, Jena, Germany) fully equipped for FCS analysis. The sample was excited by the $514 \mathrm{~nm}$ laser line and focused through a C-Apochromat 40X/1.2 NA waterimmersed objective via a dichroic mirror. The fluorescence was detected by the same objective and was spectrally divided and detected by a 32 channel GaAsP detector after passage through a pinhole in the image plane. By measurement on the dye Rhodamine 6G, using $D_{\text {Rh6G }}=$ $4.14 \cdot 10^{-10} \mathrm{~m}^{2} \mathrm{~s}^{-1},{ }^{23}$ the radius $\omega$ and the volume of the confocal detection volume were estimated to $\omega=0.23 \mu \mathrm{m}$ and $\mathrm{V}_{\mathrm{dv}, 514}=0.43 \mathrm{fL}$ respectively. FCS analysis was performed using the Zen 2012 software (Zeiss, Jena, Germany) as well as in house written functions using Origin 9.1 (Originlab Corporation, USA). 
For a sample containing particles of two different main sizes, and where translational diffusion is the only process giving rise to fluorescence fluctuations, the autocorrelation function in an FCS measurement is fitted to:

$$
G(\tau)=\frac{1}{N}\left(\frac{1-\theta}{\left(1+\frac{\tau}{\tau_{D 1}}\right)} \frac{1}{\left(1+\frac{\omega^{2} \tau}{z^{2} \tau_{D 1}}\right)^{\frac{1}{2}}}+\frac{\theta}{\left(1+\frac{\tau}{\tau_{D 2}}\right)} \frac{1}{\left(1+\frac{\omega^{2} \tau}{z^{2} \tau_{D 2}}\right)^{\frac{1}{2}}}\right)+1
$$

Here $\mathrm{N}$ is the mean total number of particles in the detection volume (i.e. the sum of both species), $\tau_{\mathrm{D} 1}$ and $\tau_{\mathrm{D} 2}$ are the diffusion times of the two main species through the detection volume, $\theta$ is the fraction of the curve resulting from species 2 , and $\omega$ and $\mathrm{z}$ denote the distances in the radial and axial dimensions respectively, at which the average detected fluorescence intensity has dropped to $\mathrm{e}^{-2}$ of its peak value. ${ }^{24}$

Transmission electron microscopy Freezing of the cryo-TEM sample was carried out using a controlled environment vitrification system keeping the relative humidity close to saturation at around $26^{\circ} \mathrm{C}$. $\mathrm{MSDH}$ was resuspended to a final concentration of $39 \mathrm{mM}$ (1.25\%) in 10 $\mathrm{mM} \mathrm{KCl,} 10 \mathrm{mM}$ HEPES at pH 7.2 or 5.5, gently vortexed and kept at $4{ }^{\circ} \mathrm{C}$ until cryo-TEM experiments were perfomed. A $5 \mu \mathrm{L}$ drop of this solution was placed on a carbon grid and the excess fluid was gently blotted away, leaving a thin film covering the grid. The grid was then rapidly vitrified by plunging into liquid ethane $\left(-180^{\circ} \mathrm{C}\right)$, and stored in liquid nitrogen until examination. The micrographs were recorded in a Philips CM120 BioTWIN Cryo with a Gatan MSC791 cooled-CCD camera, operating at $120 \mathrm{kV}$ under low-electron-dose conditions.

\section{Results and Discussion}

MSDH assembly into vesicles is $p H$-dependent, and is reversible.

At pH 7.2, large vesicles were observed in the cryo-TEM images, which showed a single bilayer delimiting (in most cases) empty compartments (Fig 2A). The shape and size of these structures was not uniform and we could observe vesicles of diameters ranging from 50 to 500 $\mathrm{nm}$. Sometimes small vesicles were observed inside larger ones. The approximate width of their limiting membrane was $30 \AA$, compatible with two opposed monolayers formed by molecules of the size of MSDH. At pH 5.5, these large vesicles were completely absent and only very small aggregates, i.e. of less than $5 \mathrm{~nm}$ diameter, were observed (Fig 2B). 

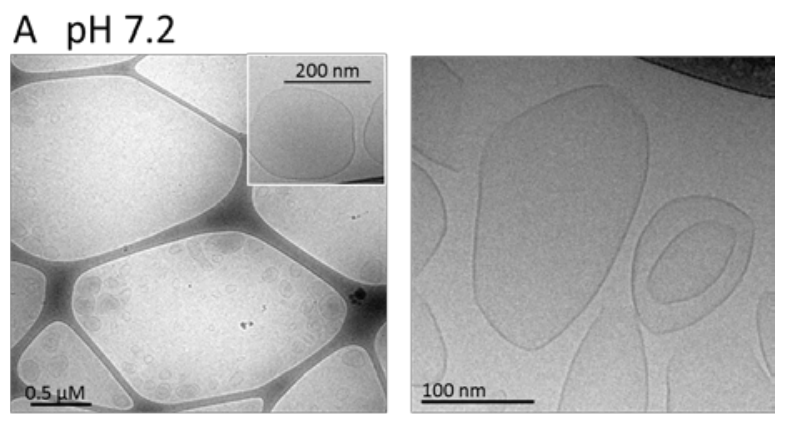

\section{B $\mathrm{pH} 5.5$}
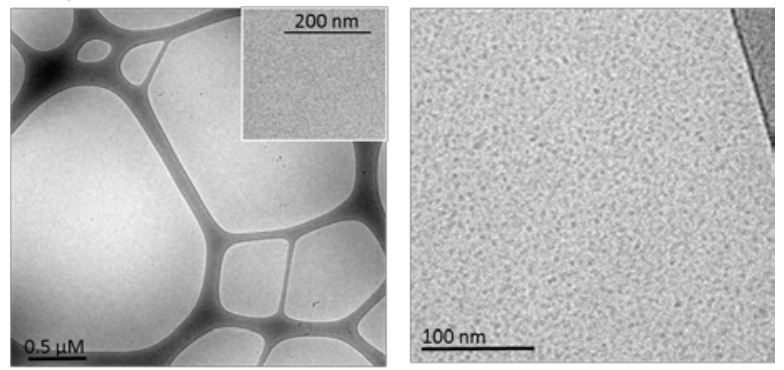

Figure 2. Cryo electron microscopy images. (A) MSDH vesicles resuspended at pH 7.2. (B) MSDH vesicles resuspended at $\mathrm{pH}$ 5.5.

We used DLS to further study the behavior of MSDH vesicles over a wider range of $\mathrm{pH}$ values. In order to ensure a homogeneous population of vesicles, a suspension of MSDH (60 $\mathrm{mM}$ ) in Milli-Q water was extruded through a $100 \mathrm{~nm}$ membrane filter. These extruded vesicles were in turn resuspended at a final MSDH concentration of $600 \mu \mathrm{M}$ in solutions of different $\mathrm{pH}$ to investigate their size and shape. MSDH vesicles resuspended at $\mathrm{pH} 6.4$ or lower spontaneously disassemble as is qualitatively shown by the time autocorrelation functions from the DLS measurements (Figure 3A). Above this $\mathrm{pH}$ the overlapping correlation curves, i.e. the constant size distribution of the vesicles, indicate that over a wide range of $\mathrm{pH}$ values MSDH vesicles retain their size, and at $\mathrm{pH}$ 7.2 extruded MSDH vesicles retain their size for at least 72 hrs (Figure S2, Supporting Information). For dilute systems, the DLS count rate varies linearly with the number of scattering particles of equal size, but varies as the sixth power of the radius of the scatterers. The steep increase in count rate from $\mathrm{pH} 6.4$, for samples of equal MSDH concentration, is thus indicative of the presence of large assemblies above this $\mathrm{pH}$ (Figure 3B). The z-averaged sizes of the assemblies, as obtained from a cumulants analysis of the correlation functions, are shown in Figure 3C. Although this 
method is much less affected by data noise than distribution analysis, we were still not able to reliably obtain sizes for $\mathrm{pH}<6.4$ (for which the noise is clearly evident from Figure 3A).

The zeta potential is a parameter correlated with particle stability because non-charged particles are less likely to remain dispersed in solution. We performed zeta potential measurements on the same systems as the DLS measurements, i.e. extruded vesicles resuspended in solutions at different $\mathrm{pH}$. Above $\mathrm{pH} 6.4$ and up to $\mathrm{pH} 11$, vesicles were found to be positively charged (Fig 3D). The highest zeta potential is found immediately upon formation of the vesicles at $\mathrm{pH}$ around 7 . The zeta potential then decreases continuously as the $\mathrm{pH}$ is increased, and turns negative at around $\mathrm{pH} 11$. The low zeta potential values obtained below pH 6.4 are most probably indicating the progressive disassembly leading to the absence of the vesicles in this $\mathrm{pH}$ range. The TEM images show small $(<5 \mathrm{~nm})$ aggregates under acidic conditions, these are most likely micelles, but our data is not conclusive on this point. Protonation of MSDH, exposing a primary amine, increases as $\mathrm{pH}$ is decreased, hence for given particle size and ionic environment, the $\zeta$-potential of MSDH aggregates would increase with a further decrease in $\mathrm{pH}$. This is also what Figure 3D shows while changing from high to low $\mathrm{pH}$, until $\mathrm{pH}$ reaches 6.4, where there is instead a sharp drop in potential. The TEM and DLS data clearly show that aggregate size is dramatically reduced at low $\mathrm{pH}$. The $\zeta$-potential does normally not vary with particle size, but with surface charge density. Hence, there is no obvious reason why, for example, micelles would have dramatically lower $\zeta$-potential than the vesicles in an otherwise similar environment. After a bilayer-to-micelle transition, where the area per headgroup is presumably larger and charge regulation via headgroup repulsion decreases, even an increase in surface charge density could be expected. However, there might be a technical reason why this is not observed; the stated lower diameter limit for electrophoretical light scattering with the Zetasizer Nano ZS90 is $3.8 \mathrm{~nm}$, and we suggest that the sharp drop in $\zeta$-potential at $\mathrm{pH}$ below 6.4 is a result of reduction in aggregate size outside of the available range of the Zetasiser. This does not imply that the aggregates are smaller than $3.8 \mathrm{~nm}$, since the lower useful range is sample-dependent, and could in reality be greater. Hence, from our data, it is not possible to present a consistent picture of the structure of the aggregates at low $\mathrm{pH}$. 
A

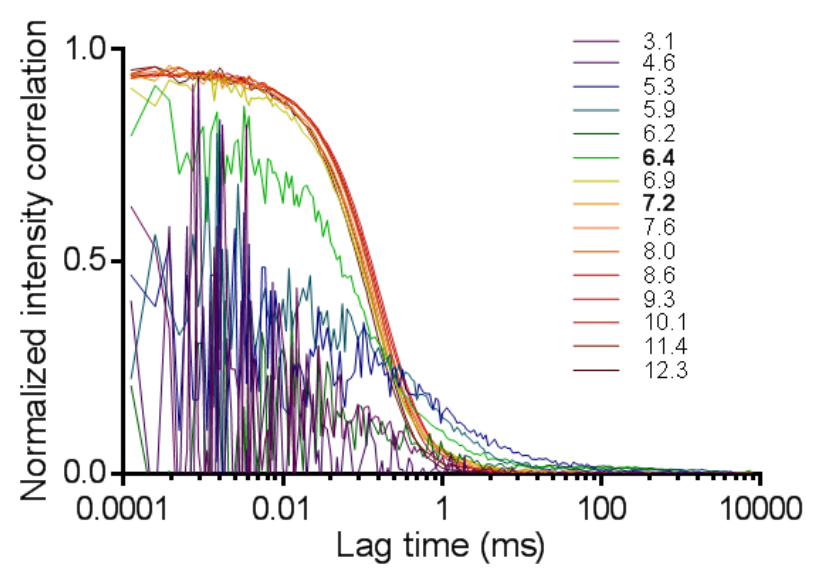

B
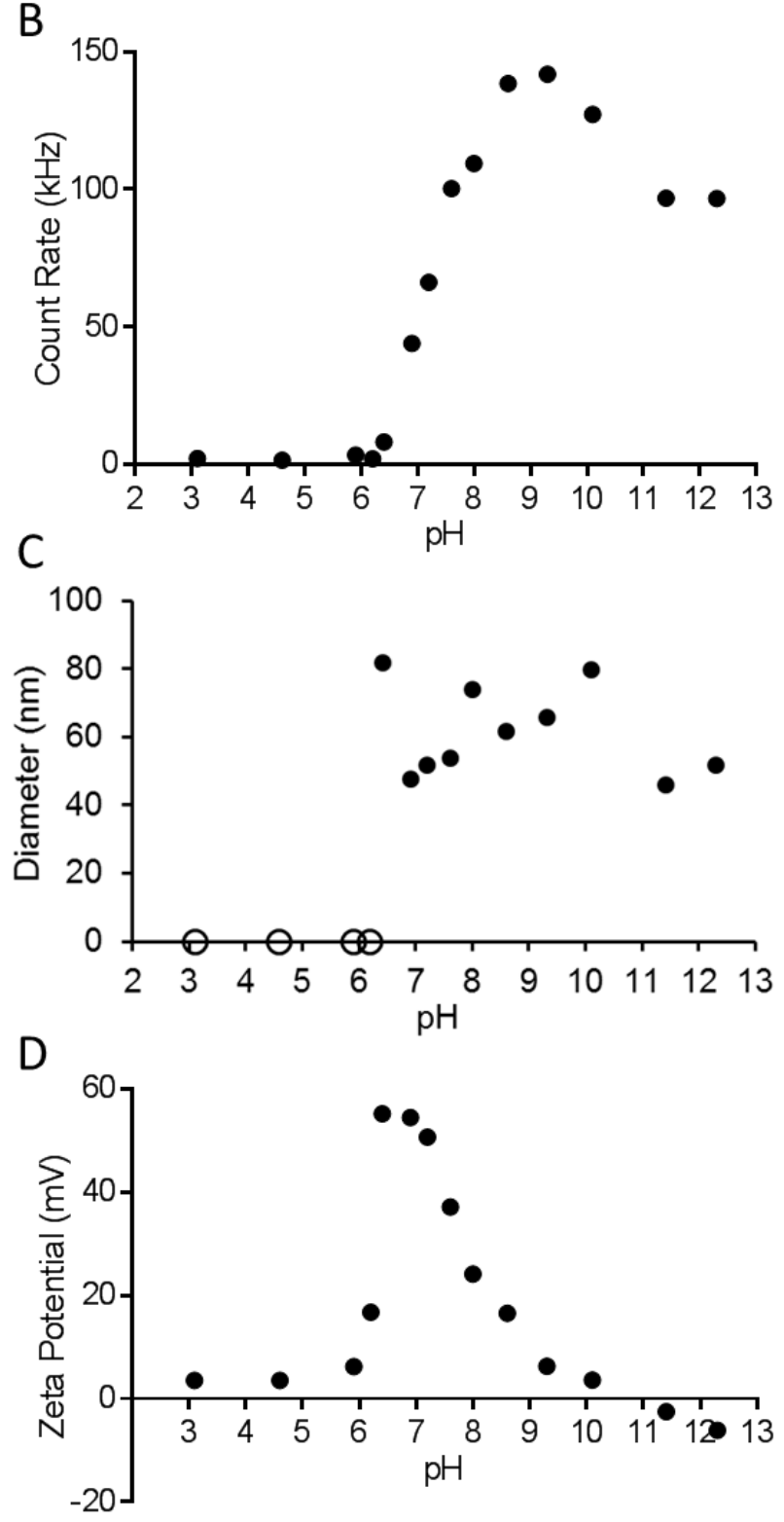
Figure 3. Dynamic light scattering of MSDH vesicles extruded through $100 \mathrm{~nm}$ membrane pores. The data in this figure was assembled from four independent experiments. (A) Autocorrelation functions measured for MSDH vesicles resuspended in solutions of different $\mathrm{pH}$ values. Curves for $\mathrm{pH}>7.2$ overlap (a larger version of the graph, where the curves can be more easily distinguished, is included as Figure S1 in Supporting Information). (B) Count rate values obtained together with the curves in Figure 3A. (C) The z-average hydrodynamic diameters for the data in shown in Figures 3A and 3B. For low values of the $\mathrm{pH}$ (indicated by circles), the radii could not be unequivocally determined due to the low intensity and high S/N ratio. The polydispersity index for $\mathrm{pH} 6.4$ is 0.37 , and falls in the range $0.18-0.26$ for the other radii. (D) Zeta potential of MSDH vesicles resuspended in buffers of different $\mathrm{pH}$.

The reversibility of the $\mathrm{pH}$-dependent aggregation was tested by cycling the $\mathrm{pH}$ in a solution of MSDH by alternate addition of $\mathrm{HCl}$ and $\mathrm{NaOH}$. The result of such a test for a vesicle solution where the $\mathrm{pH}$ was twice cycled from low to high $\mathrm{pH}$, and back to low again, is shown in Figure 4. Since the MSDH concentration remains constant (not accounting for successive dilution of the sample, which amounts to ca. $5 \%$ for the entire series), the variations in scattering intensity relate to variations in the sizes of the aggregates.

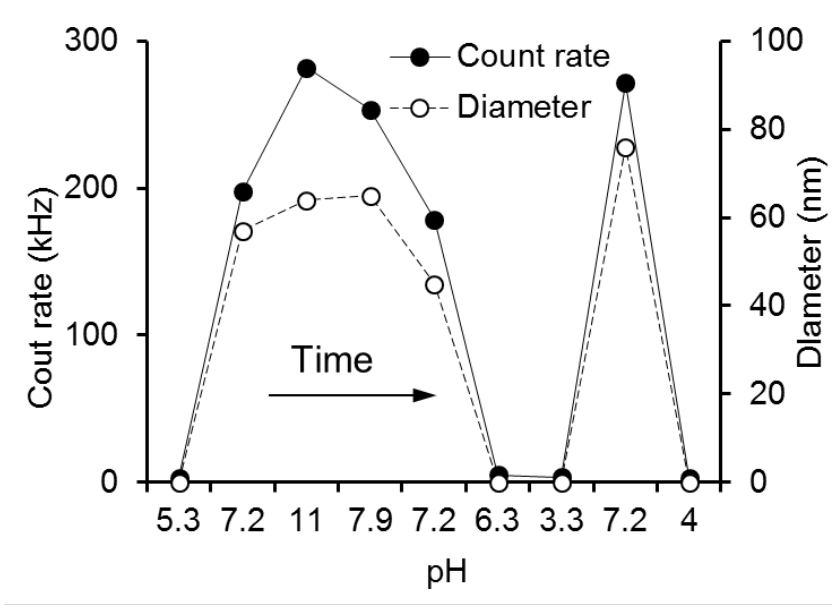

Figure 4. Dynamic light scattering count rates and z-average diameters for a vesicle solution where the $\mathrm{pH}$ is altered sequentially from low to high $\mathrm{pH}$ and back again, in two cycles, by addition of $\mathrm{HCl}$ or $\mathrm{NaOH}$. Measurements were obtained from left to right. 
MSDH vesicles can be efficiently loaded at neutral $\mathrm{pH}$, and release of the contents triggered by low $\mathrm{pH}$.

In order to verify that MSDH forms vesicles containing an interior volume, and to evaluate whether it is possible to load MSDH vesicles, we used the auto-quenching properties of CXF, which is routinely used to assess encapsulation and release of this fluorophore (Figure 5). MSDH vesicles were loaded with large amounts of CXF by making use of MSDH differential behavior at low and high $\mathrm{pH}$, i.e. resuspending $\mathrm{MSDH}$ in a buffer of low $\mathrm{pH}$ where it is not forming vesicles and triggering the formation of vesicles in the presence of CXF by raising the $\mathrm{pH}$. Excess CXF not encapsulated in vesicles was removed by gel filtration. The increased fluorescence emission upon addition of Triton X-100 indicates that upon disruption of the vesicles the fluorophore is released into a larger volume in which it is no longer autoquenched (Figure 5A). Note that CXF was not loaded into the vesicles upon hydration of the MSDH, which is the customary and more efficient way of loading fluorophores into lipid vesicles for leakage assays, but via re-formation of vesicles from MSDH already in solution. This method was used to confirm that re-assembly into vesicles is $\mathrm{pH}$-induced and can be performed in bulk solution, but results in comparatively lower quantities of entrapped fluorophores, and hence a less effective quenching. The fluorescence emission in Figure 5A remains stable for vesicles tested several days after preparation, but due to the low encapsulation efficiency, small changes related to slow leakage of the fluorophore would be difficult to detect in the assay.

The same encapsulation procedure was followed to load MSDH vesicles with Rhodamine 6G to a final concentration of $1 \mu \mathrm{M}$ inside the vesicles for FCS measurements. The fluorescence emission of Rhodamine 6G is not affected by $\mathrm{pH}$, unlike that of CXF. Using FCS we assessed the diffusion rate of Rhodamine 6G when vesicles were resuspended in a buffer at $\mathrm{pH} 7.2$ or at pH 4 (Figure 5B). A dual diffusion component model was fitted to the experimental data and the decay times of the curves are summarized in Table 1. At pH 7 a slow diffusion component corresponding to the diffusion of MSDH vesicles was present in the FCS curve, in addition to a fast component which indicates the diffusion of free Rhodamime 6G and of small aggregates. At pH 4, and also at pH 7 with Triton X-100 added, the diffusion time of the slow component was reduced substantially, indicating disruption of the MSDH vesicles (Figure 5B and Table 1). 


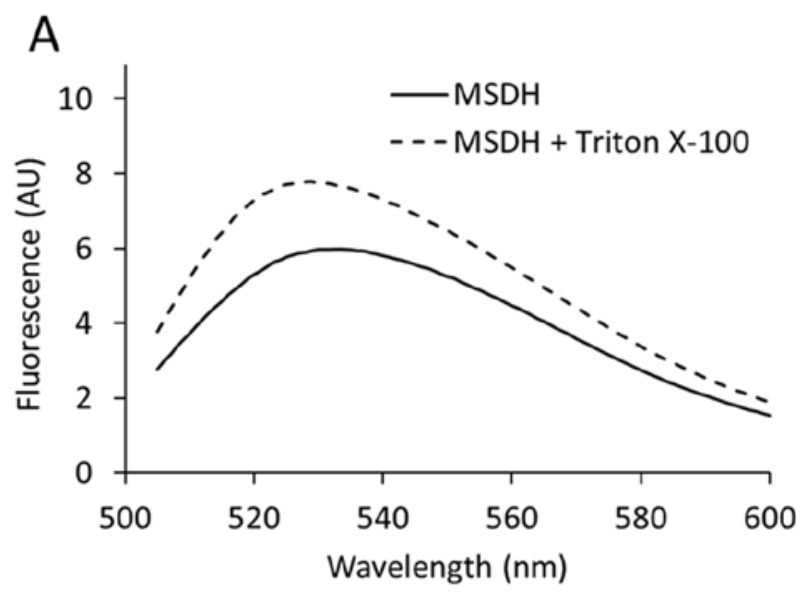

B

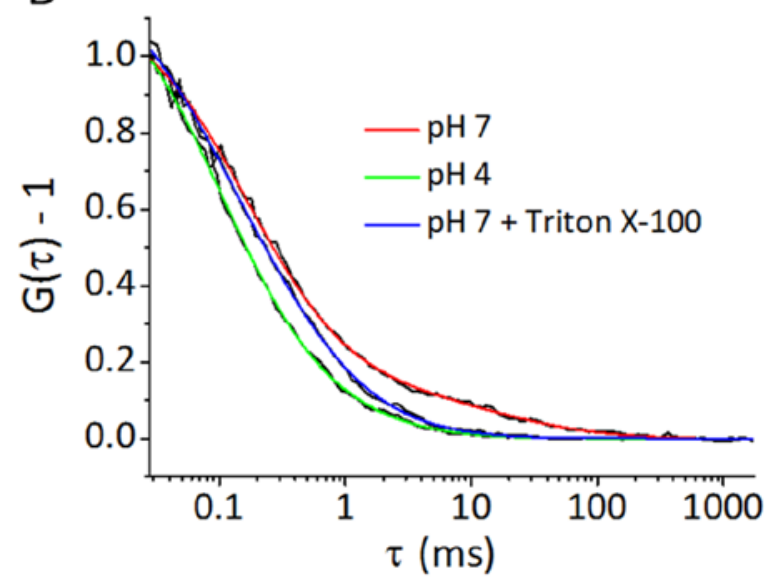

Figure 5. Fluorophore encapsulation capacity of MSDH vesicles. (A) Fluorescence emission of (self-quenching) carboxyfluorescein encapsulated in MSDH vesicles before and after the addition of Triton X-100. Vesicles were loaded by $\mathrm{pH}$-induced formation of vesicles in a bulk solution containing carboxyfluorescein and MSDH. Fluorescence was measured at pH 7.2.

(B) Normalized autocorrelation data from FCS measurements of Rhodamine 6G encapsulated in $\mathrm{MSDH}$ vesicles at $\mathrm{pH} 7.2$, at $\mathrm{pH} 4$, and after the addition of Triton $\mathrm{X}-100$ to vesicles at $\mathrm{pH}$ 7. Data in black lines, coloured lines are fits using a two-component diffusion model.

Table 1. Decay times and relative amplitudes for the autocorrelation of Rhodamine 6G in intact ( $\mathrm{pH} 7.2$ ) or disrupted ( $\mathrm{pH} 4$ or $\mathrm{pH} 7+$ Triton $\mathrm{X}-100)$ MSDH vesicles. The errors are standard errors obtained from the routine used to fit the model to the data.

\begin{tabular}{|c|c|c|c|c|}
\hline Sample & $\tau_{\mathrm{D} 1}(\mu \mathrm{s})$ & $\mathrm{a}_{1}(\%)$ & $\tau_{\mathrm{D} 2}(\mu \mathrm{s})$ & $\mathrm{a} 2(\%)$ \\
\hline pH 7 & $174 \pm 6$ & $91 \pm 0.5$ & $19000 \pm 3000$ & $9 \pm 0.5$ \\
\hline
\end{tabular}




\begin{tabular}{|c|c|c|c|c|}
\hline pH 4 & $101 \pm 5$ & $96 \pm 2$ & $2500 \pm 700$ & $4 \pm 2$ \\
\hline pH 7 + Triton X-100 & $90 \pm 15$ & $69 \pm 8$ & $550 \pm 15$ & $31 \pm 8$ \\
\hline
\end{tabular}

\section{MSDH aggregate structure}

The driving force for self-assembly of amphiphiles into organized structures is the gain in free energy resulting from a reduced contact area between water and the hydrophobic regions of the molecules ${ }^{25}$. The final shape of the assembled structure is mainly dictated by geometrical constraints that have been formalized in the concept of packing parameters ${ }^{26}$. Thus, singletailed amphiphiles usually assemble into micelles because the lateral area of their headgroup is large relative to the volume filled by their hydrophobic chain. However, under certain conditions, they can also assemble into vesicles, i.e., topologically closed structures with one or several bilayers enclosing an aqueous core. Vesicular assemblies are explored for a wide range of applications, such as cell mimicking ${ }^{27}$, drug delivery ${ }^{28}$, nano medicine ${ }^{29}$ or as chemical reactors ${ }^{30}$, and considerable effort has been invested in controlling their formation and properties. Phospholipid vesicles are well established as model systems across a number of fields ${ }^{31}$, but also gemini surfactants ${ }^{32,33,34}$, catanionic ${ }^{35,36}$ or other binary amphiphile systems ${ }^{37,} 38$, 39 and indeed also some single-tailed amphiphiles $40,41,42,43,44,45$ have been shown to form vesicles.

MSDH aggregates under neutral $\mathrm{pH}$, as revealed in the cryo-TEM images, are mostly unilamellar, or sometimes oligolamellar vesicles, with rarely more than 3 nested bilayers. The wall thickness of the layer enclosing the structures is estimated to approximately $30 \AA$, which is consistent with the thickness of two MSDH layers with not completely stretched hydrocarbon chains, hence supporting the conclusion that MSDH forms bilayers. The aggregates are mostly rounded and closed, sometimes with pores or open sides, and occasionally also with sharp bends. While some of these features might be artefacts from the vitrification of the sample, or remnants of perforated vesicles, in either case it indicates a bilayer with relatively low rigidity. The DLS results show that aggregates are $\mathrm{pH}$ sensitive, and that there is a sharp transition in aggregate size around $\mathrm{pH}$ 6.4. Aggregates formed by extrusion through $100 \mathrm{~nm}$ membrane pores retain a z-averaged diameter of 50-80 nm at, and above, neutral pH. Both the CXF leakage assay and the FCS measurements demonstrate that under neutral conditions, the MSDH aggregates are closed vesicles, and contain an interior volume, into which molecules can be entrapped. 


\section{Implications for lysosomotropism}

MSDH has been used as a lysosomotropic compound to trigger apoptotic cell death in a number of cellular systems. ${ }^{10,19}$ The generally accepted mode of action for MSDH, like for other lysosomotropic detergents, ${ }^{2,3,4}$ is that as a weakly basic amphiphilic molecule, it is uncharged at the $\mathrm{pH}$ in the cytosol, and diffuses freely across cellular membranes until it reaches the lysosomes, which are the only compartments where the $\mathrm{pH}$ is low enough for MSDH to become protonated. After protonation in the lysosome, MSDH is believed to be trapped due to the reduced diffusion across the membrane, therefore accumulating, and eventually being able to disrupt the lysosomal membrane from the interior of the lysosomal compartment. Our study is the first addressing the physicochemical properties of MSDH and its aggregates, and it presents a series of results which are at variance with this commonly held view. MSDH is non-toxic to cells when used in concentrations below 7.5 $\mu \mathrm{M}$ (Ghosh et al. ${ }^{20}$ and unpublished results $\mathrm{KÖ}$ ) and the concentrations that are used to cause cell toxicity are typically 15-25 $\mu \mathrm{M}^{10,18,19}$ DLS experiments at successively lower concentrations were carried out to establish the onset of aggregation. Size distributions at concentrations as low as $10 \mu \mathrm{M}$ still reveal large aggregates, see Figure 6. Upon addition of Triton X-100, these rapidly disintegrate into much smaller aggregates by solubilization. This demonstrates that MSDH forms aggregates also at the concentrations used in cell studies. Protonation at lower $\mathrm{pH}$ tends to increase the critical aggregation concentration (CAC), but our preliminary data indicates that at 6.4, the CAC is still $<40 \mu \mathrm{m}$ over a wide range of salinities. (Detailed investigations of the concentration dependence on aggregation at different $\mathrm{pH}$ values are in progress, and will be reported elsewhere).

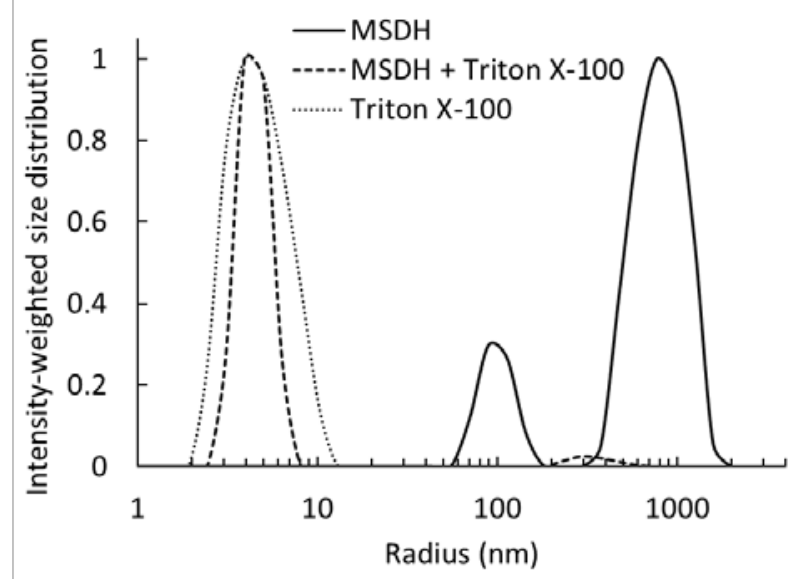

Figure 6. Normalized intensity-weighted size distributions for MSDH at $10 \mu \mathrm{M}$ in a HEPES buffer at $\mathrm{pH} 7.2$ and after solubilization of the MSDH by addition of Triton X-100. For 
comparison, the sizes of aggregates formed by Triton X-100 are included, too. Note that scattered intensity scales with $r^{6}$, and intensity-weighted size distributions thus give disproportionate weight to larger particles. The total intensity contributing to the peak centered round $r \approx 1 \mu \mathrm{m}$ for MSDH is $5 \times$ greater than that for $r \approx 100 \mathrm{~nm}$, but this still corresponds to a much greater number of small aggregates (by approximately $200 \times$ ).

Consequently, the observation that at neutral $\mathrm{pH}$, and already at $10 \mu \mathrm{M}$ concentration (Figure 6), MSDH is spontaneously assembling into vesicles with large diameters, suggest that, rather than entering the cell as monomers that diffuse across the plasma membrane, other possibilities must be considered. With MSDH forming aggregates under cytosolic (and indeed also intercellular) conditions, it is, in principle, still possible that MSDH enters through the plasma and lysosomal membranes as monomers via disassembly and passive diffusion. However, this appears unlikely; first, an important prerequisite of the free diffusion across membranes and subsequent retention of LDs in the lysosomes, is the presumed lack of detergent properties of LDs in the cytosol. Our data invalidates this presumption, and with MSDH itself forming bilayers, it is difficult to see why MSDH would not partition into any other lipid structures available in the cell. Second, and most importantly, the zeta potential data (Figure 3D) shows that the MSDH aggregates are positively charged at cytosolic $\mathrm{pH}$. Cationic liposomes have been shown to traverse plasma membranes effectively, and are used as efficient drug delivery vehicles. Since the pioneering work on DNA transfection by Felgner ${ }^{46}$ this phenomenon has been utilized mostly for gene delivery ${ }^{47}$, but increasingly also for delivery of small molecules ${ }^{48}$. A recent study found that cationic liposomes were readily taken up by cells, and traversed the plasma membrane via some endocytotic pathway, without adverse effects on the cell. ${ }^{49}$ Based on the data presented here, the previous literature on MSDH action and effects on cells, as well as the extensive literature on cationic liposome uptake, we propose that MSDH could be taken up by an endocytic mechanism. As lysosomes are the last compartments in the endocytic route, MSDH vesicles would be precisely targeted to these organelles. According to our results, once the $\mathrm{pH}$ has dropped below 6.4, MSDH vesicles will disassemble into small aggregates or dissolve completely. While the literature on the solubilization of lipid membranes by detergents is extensive and addressing several aspects of solubilization or permeabilization of bilayers by detergents ${ }^{50}$, the interaction of detergents with membranes under the conditions particular to lysosomes appears not to have been investigated, and this is the subject of current studies in our laboratories. 
pH-dependence

Tuning the structure of self-assembled molecular aggregates requires control of parameters affecting aggregate structure, such as headgroup polarity and effective area, or hydrophobic chain length or volume. In this vein, much of the efforts aiming at vesiculation of surfactants has used binary surfactant systems to achieve good control of, and to balance, these parameters. However, contrary to this, it has been shown that a rich phase behaviour with structural transformations do not require chemically sophisticated amphiphiles, but can be obtained with simple molecules such as alkyl diamines. ${ }^{51,52}$ An essential requisite for this is the $\mathrm{pH}$-sensitivity of amines, and in particular for diamines (and thus potentially also for $\mathrm{MSDH}$ ) that the differences in $\mathrm{pK}_{\mathrm{a}}$ between the amine moieties can be exploited. Protonated and deprotonated molecules will vary in polarity, perhaps also in conformation, and interact differently with the environment. Thus, there will be a $\mathrm{pH}$ range where a solution of singlecomponent $\mathrm{pH}$-sensitive surfactants behaves like a binary mixture of the protonated and deprotonated forms, and where solution $\mathrm{pH}$ is used to tune the composition. ${ }^{53}$ Protonation of the MSDH headgroup at $\mathrm{pH}<6.4$ either increases solubility so that the critical aggregation concentration is increased and any aggregates dissolved, or increases headgroup repulsion enough to transform the vesicles to micelles, or other aggregates much smaller than vesicles. The former result is in agreement with the observations by Dubowchik et al., ${ }^{4}$ that on going from $\mathrm{pH} 4.8$ to $\mathrm{pH} 7.0$, there was a considerable loss of solubilization capacity of MSDH. Our TEM images, on the other hand, indicate the presence of small aggregates, but conditions were not identical. The repeated assembly and disassembly of large MSDH aggregates, upon cycling the $\mathrm{pH}$, shows that aggregation and disassembly of the aggregates is fully controllable via solution $\mathrm{pH}$. The encapsulation of fluorophores from bulk solutions, as demonstrated in the leakage assay and the FCS results, show that this aggregation process results in closed, vesicular structures, in agreement with the TEM data. The coexistence of protonated and deprotonated forms of MSDH will persist over a certain $\mathrm{pH}$ range, and at extreme $\mathrm{pH}$ values, the solution will act as a single-component system. It is likely that the size or structure of the aggregates varies with $\mathrm{pH}$, even at $\mathrm{pH}>7.2$, as suggested by the data at high $\mathrm{pH}$ in Figure $3 \mathrm{~B}$. It is possible that the stabilization of the count rate around $100 \mathrm{kHz}$ represents the end of the coexistence region, where the deprotonation is saturated, but the data does not extend far enough to allow a verification of this. 
$\mathrm{pH}$-responsive materials in general, and vesicles in particular, are of interest for potential applications in drug delivery. ${ }^{54,55}$ The $\mathrm{pH}$ of the organelles in the endolysosomal pathway decreases successively, from the early endosomes ( $\mathrm{pH}$ 6.5), via late endosomes, to the lysosomes ( $\mathrm{pH}$ 3.5-5), and as the last step in this pathway, lysosomes are good candidates for targeted delivery. In the field of nano medicine, pH-responsive lipid-based vesicles were recognized as promising delivery tools long ago ${ }^{56,57}$ and $\mathrm{pH}$-dependent liposome fusion was considered early $\mathrm{on}^{58}$. In more recent years, several groups have tried to develop $\mathrm{pH}$ responsive nano-carriers using different building blocks. ${ }^{59,60,61}$ Much of the interest in $\mathrm{pH}$ triggered drug release stems from the need to effectively target microenvironments with intrinsically low $\mathrm{pH}$, such as endosomes or extracellular tumor environments ${ }^{55}$, and for the use of $\mathrm{pH}$-responsive vehicles for gene delivery ${ }^{54}$. For therapeutic purposes, rapid drug release upon arrival to the pathological tissue is highly desired, since this will maximize the therapeutic efficacy, and also minimize the development of drug resistance. Polymeric systems, such as block co-polymers ${ }^{62}$ or dendrimers ${ }^{63}$ are often used to form carrier systems since the greater freedom in the choice of chemistry and molecular size gives better control of stability and retention of encapsulated matter, but at the cost of slower kinetics, and increased difficulties in adjusting the permeability for rapid release or uptake of molecules. Many polymer-based vesicles (polymerosomes), relying on diffusion of active substances across the polymer wall do not meet the requirement for rapid release and respond slowly to external stimuli; hence there is still considerable interest in designing $\mathrm{pH}$-responsive surfactant-based systems allowing for rapid disassembly and release. Routes for directed delivery to particular organelles have been extensively investigated, ${ }^{64}$ and strategies adopted specifically for lysosomal delivery also include $\mathrm{pH}$-responsive nanogels, ${ }^{65}$ polyelectrolyte complexes, ${ }^{66}$ or acid-sensitive micelles. ${ }^{67}$ However, this issue has mostly been adressed by covalent linking of lysosomotropic ligands either to liposomes ${ }^{68,69}$ or directly to active substances ${ }^{70,71,72}$, often with limited lysosomal specificity of the cargo-carrier conjugate.

We demonstrate, via the $\mathrm{pH}$-dependent disassembly, that MSDH forms vesicles which are capable of containing, and, at a suitable $\mathrm{pH}$, release its contents. We show that this can be done both using high concentrations of cargo, as concluded from the carboxyfluorescein autoquenching data, and low concentrations of cargo, as concluded from the fluorescence correlation spectroscopy data. In the latter, the slow diffusion component at $\mathrm{pH} 7$ indicates the presence of MSDH vesicles encapsulating Rhodamine 6G, while at $\mathrm{pH} 4$ the diffusion time of 
the slow component is substantially reduced, indicating that Rhodamine 6G is released after disassembly of the vesicles. At $\mathrm{pH} 7$ with the addition of Triton X-100 the slow diffusion time is also reduced, but the fraction of the slow component is larger (Table 1). This may be the result of solubilization of Rhodamine 6G in mixed aggregates of MSDH and Triton X-100. Taken together, these results demonstrate that MSDH vesicles can efficiently encapsulate and retain small molecules that are released when MSDH vesicles are disrupted at sufficiently low $\mathrm{pH}$. As such, we propose that MSDH has potential for use in $\mathrm{pH}$-triggered lysosomal drug delivery applications.

It is of considerable practical interest that our physicochemical studies, which clarify the aggregation properties of MSDH under conditions in the cytosol and inside lysosomes, also reveal a possible path for targeted and specific delivery to lysosomal compartments. Cell studies unequivocally demonstrate that MSDH induces lysosomal membrane permeabilization, and there is an MSDH dose range between the onset of aggregation and the toxicity concentration, where MSDH still accumulates in the lysosomes. We propose that this offers a window which could potentially be explored for endocytic delivery of MSDHencapsulated cargo, for subsequent pH-triggered release in the interior of the lysosomes, without release of the cargo to the cytosol.

\section{Summary and Conclusions}

We have initiated a study with a view to understand the mechanism of action of the lysosomotropic detergent MSDH. As a first step, we have considered the $\mathrm{pH}$-dependent aggregation of MSDH itself, and some of our results conflict with the dominating picture of how LDs reach the lysosomal compartment. We have found that MSDH spontaneously forms vesicles near, or above, cytosolic $\mathrm{pH}$, and at very low concentrations $(<10 \mu \mathrm{M})$, suggesting that endocytic transport to the lysosomes is probable. However, this will need to be confirmed by cell studies. Further, we demonstrate that MSDH vesicles can be loaded with small molecules via encapsulation induced via $\mathrm{pH}$ changes. The release of the contents can be triggered via Triton X-100 detergent solubilization, or via lowering of the $\mathrm{pH}$, and hence MSDH could be of interest for delivery systems with $\mathrm{pH}$-triggered release of molecules.

\section{Acknowledgements}

We thank Gunnel Karlsson for skilled assistance during the cryo-TEM experiments. This work was financially supported by the Swedish Research Council (grant 2013-3214 to KÖ) 
and Konung Gustaf V och Drottning Victorias Frimurarestiftelse (KÖ) and Stiftelseförvaltningen Region Östergötland (grant LIO-570261 to AMVG). TE acknowledges financial support from the Swedish Government Strategic Research Area in Materials Science on Functional Materials at Linköping University (Faculty Grant SFO-Mat-LiU \#2009-00971). ANP acknowledges support from Linköping University through Rector’s Guest professorship Programme.

\section{Supporting Information}

Supporting information available: Magnified reproduction of Figure 3A, and DLS data demonstrating the stability of MSDH vesicles. 


\section{References}

1. Villamil Giraldo, Ana M.; Appelqvist, H.; Ederth, T.; Öllinger, K. Lysosomotropic agents: impact on lysosomal membrane permeabilization and cell death. Biochem. Soc. Trans. 2014, 42 (5), 1460-1464.

2. De Duve, C.; De Barsy, T.; Poole, B.; Trouet, A.; Tulkens, P.; Van Hoof, F. Lysosomotropic agents. Biochem. Pharmacol. 1974, 23 (18), 2495-2531.

3. Firestone, R. A.; Pisano, J. M.; Bonney, R. J. Lysosomotropic agents. 1. Synthesis and cytotoxic action of lysosomotropic detergents. J. Med. Chem. 1979, 22 (9), 1130-1133.

4. Dubowchik, G. M.; Gawlak, S. L.; Firestone, R. A. The in-vitro effects of three lysosomotropic detergents against three human tumor cell lines. Bioorg. Med. Chem. Lett. 1995, 5 (8), 893-898.

5. Miller, D. K.; Griffiths, E.; Lenard, J.; Firestone, R. A. Cell killing by lysosomotropic detergents. J. Cell. Biol. 1983, 97 (6), 1841-1851.

6. Forster, S.; Scarlett, L.; Lloyd, J. B. The effect of lysosomotropic detergents on the permeability properties of the lysosome membrane. Biochim. Biophys. Acta 1987, 924 (3), 452-457.

7. Wilson, P. D.; Hreniuk, D.; Lenard, J. Reduced cyto-toxicity of the lysosomotropic detergent n-dodecylimidazole after differentiation of hl60 promyelocytes. Cancer Research 1989, 49 (3), 507-510.

8. Boyer, M. J.; Horn, I.; Firestone, R. A.; Steele-Norwood, D.; Tannock, I. F. pH dependent cytotoxicity of N-dodecylimidazole: a compound that acquires detergent properties under acidic conditions. Br J Cancer 1993, 67 (1), 81-87.

9. Cabantchik, Z. I.; Silfen, J.; Firestone, R. A.; Krugliak, M.; Nissani, E.; Ginsburg, H. Effects of lysosomotropic detergents on the human malarial parasite Plasmodium falciparum in in vitro culture. Biochem. Pharmacol. 1989, 38 (8), 1271-1277.

10. Li, W.; Yuan, X. M.; Nordgren, G.; Dalen, H.; Dubowchik, G. M.; Firestone, R. A.; Brunk, U. T. Induction of cell death by the lysosomotropic detergent MSDH. FEBS Lett. 2000, 470 (1), 35-39.

11. Ostenfeld, M. S.; Hoyer-Hansen, M.; Bastholm, L.; Fehrenbacher, N.; Olsen, O. D.; Groth-Pedersen, L.; Puustinen, P.; Kirkegaard-Sorensen, T.; Nylandsted, J.; Farkas, T.; Jaattela, M. Anti-cancer agent siramesine is a lysosomotropic detergent that induces cytoprotective autophagosome accumulation. Autophagy 2008, 4 (4), 487-499.

12. Hussain, M.; Leibowitz, M. J.; Lenard, J. Killing of saccharomyces-cerevisiae by the lysosomotropic detergent N-dodecylimidazole. Antimicrob. Agents Ch. 1987, 31 (4), 512517.

13. Firestone, R. A.; Pisano, J. M.; Garrity, G. M.; Fromtling, R. A.; Zimmerman, S. B. Lysosomotropic agents. 7. Broad-spectrum antifungal activity of lysosomotropic detergents. J. Med. Chem. 1987, 30 (8), 1519-1521.

14. Forster, S.; Scarlett, L.; Lloyd, J. B. Effects of a lysosomotropic detergent on cystinotic fibroblasts. Biochem. Soc. Trans. 1988, 16 (1), 43-44.

15. Parry, M. J.; Alakoskela, J.-M. I.; Khandelia, H.; Kumar, S. A.; Jäättelä, M.; Mahalka, A. K.; Kinnunen, P. K. J. High-Affinity Small Molecule-Phospholipid Complex Formation: Binding of Siramesine to Phosphatidic Acid. J. Am. Chem. Soc. 2008, 130 (39), 1295312960.

16. Wilson, P. D.; Firestone, R. A.; Lenard, J. The role of lysosomal-enzymes in killing of mammalian-cells by the lysosomotropic detergent $N$-dodecylimidazole. J. Cell. Biol. 1987, 104 (5), 1223-1229.

17. Sasaki, H.; Arai, H.; Cocco, M. J.; White, S. H. pH Dependence of Sphingosine Aggregation. Biophys. J. 2009, 96 (7), 2727-2733. 
18. van Nierop, K.; Muller, F. J. M.; Stap, J.; Van Noorden, C. J. F.; van Eijk, M.; de Groot, C. Lysosomal Destabilization Contributes to Apoptosis of Germinal Center Blymphocytes. J. Histochem. Cytochem. 2006, 54 (12), 1425-1435.

19. Appelqvist, H.; Nilsson, C.; Garner, B.; Brown, A. J.; Kågedal, K.; Öllinger, K. Attenuation of the Lysosomal Death Pathway by Lysosomal Cholesterol Accumulation. Am. J. Pathol. 2011, 178 (2), 629-639.

20. Ghosh, M.; Carlsson, F.; Laskar, A.; Yuan, X.-M.; Li, W. Lysosomal membrane permeabilization causes oxidative stress and ferritin induction in macrophages. FEBS Lett. 2011, 585 (4), 623-629.

21. Ofori, L. O.; Withana, N. P.; Prestwood, T. R.; Verdoes, M.; Brady, J. J.; Winslow, M. M.; Sorger, J.; Bogyo, M. Design of Protease Activated Optical Contrast Agents That Exploit a Latent Lysosomotropic Effect for Use in Fluorescence-Guided Surgery. ACS Chem. Biol. 2015, 10 (9), 1977-1988.

22. Huth, U.; Wieschollek, A.; Garini, Y.; Schubert, R.; Peschka-Süss, R. Fourier transformed spectral bio-imaging for studying the intracellular fate of liposomes. Cytometry, Part A 2004, 57A (1), 10-21.

23. Culbertson, C. T.; Jacobson, S. C.; Michael Ramsey, J. Diffusion coefficient measurements in microfluidic devices. Talanta 2002, 56 (2), 365-373.

24. Rigler, R.; Mets, Ü.; Widengren, J.; Kask, P. Fluorescence correlation spectroscopy with high count rate and low background: analysis of translational diffusion. European Biophysics Journal 1993, 22 (3), 169-175.

25. Tanford, C. The Hydrophobic effect: formation of micelles and biological membranes; 2 ed.; Wiley: New York, 1980.

26. Israelachvilli, J. N. Intermolecular and surface forces. 1991.

27. Brüggemann, D.; Frohnmayer, J. P.; Spatz, J. P. Model systems for studying cell adhesion and biomimetic actin networks. Beilstein J. Nanotechnol. 2014, 5, 1193-1202.

28. Simão, A. M. S.; Bolean, M.; Cury, T. A. C.; Stabeli, R. G.; Itri, R.; Ciancaglini, P. Liposomal systems as carriers for bioactive compounds. Biophys. Rev. 2015, 7 (4), 391397.

29. Cabral, H.; Miyata, K.; Kishimura, A. Nanodevices for studying nano-pathophysiology. Adv. Drug Delivery Rev. 2014, 74, 35-52.

30. Dong, R.; Liu, W.; Hao, J. Soft Vesicles in the Synthesis of Hard Materials. Acc. Chem. Res. 2012, 45 (4), 504-513.

31. Chan, Y.-H. M.; Boxer, S. G. Model membrane systems and their applications. Curr. Opin. Chem. Biol. 2007, 11 (6), 581-587.

32. Liang, Z.; Wang, C.; Huang, J. The research on the vesicle formation and transformation in novel Gemini surfactant systems. Colloids Surf., A 2003, 224 (1-3), 213-220.

33. Menger, F. M.; Littau, C. A. Gemini surfactants: a new class of self-assembling molecules. J. Am. Chem. Soc. 1993, 115 (22), 10083-10090.

34. Zana, R.; Talmon, Y. Dependence of aggregate morphology on structure of dimeric surfactants. Nature 1993, 362 (6417), 228-230.

35. Kaler, E. W.; Murthy, A. K.; Rodriguez, B. E.; Joseph, A. N. Z. Spontaneous Vesicle Formation in Aqueous Mixtures of Single-Tailed Surfactants. Science 1989, 245 (4924), 1371-1374.

36. Dew, N.; Edwards, K.; Eriksson, J.; Edsman, K.; Björk, E. Gel formulations containing catanionic vesicles composed of alprenolol and SDS: Effects of drug release and skin penetration on aggregate structure. Colloids Surf., B 2012, 89, 53-60.

37. Viseu, M. I.; Edwards, K.; Campos, C. S.; Costa, S. M. B. Spontaneous Vesicles Formed in Aqueous Mixtures of Two Cationic Amphiphiles. Langmuir 2000, 16 (5), 2105-2114. 
38. Du, N.; Song, S.-E.; Hou, W.-G. Vesicle stability in aqueous mixtures of zwitterionic/anionic surfactants. Colloids Surf., A 2008, 312 (2-3), 104-112.

39. Weiss, T. M.; Narayanan, T.; Gradzielski, M. Dynamics of Spontaneous Vesicle Formation in Fluorocarbon and Hydrocarbon Surfactant Mixtures. Langmuir 2008, 24 (8), 3759-3766.

40. Hargreaves, W. R.; Deamer, D. W. Liposomes from ionic, single-chain amphiphiles. Biochemistry 1978, 17 (18), 3759-3768.

41. Kunitake, T.; Okahata, Y.; Shimomura, M.; Yasunami, S.; Takarabe, K. Formation of stable bilayer assemblies in water from single-chain amphiphiles. Relationship between the amphiphile structure and the aggregate morphology. J. Am. Chem. Soc. 1981, 103 (18), 5401-5413.

42. Imae, T.; Trend, B. Video-enhanced differential interference contrast microscope observation of $\mathrm{pH}$-dependent vesicle formation by single-chain surfactants. Langmuir 1991, 7 (4), 643-646.

43. Du, N.; Song, R.; Zhu, X.; Hou, W.; Li, H.; Zhang, R. Vesicles composed of one simple single-tailed surfactant. Chem. Comm. 2014, 50 (73), 10573-10576.

44. Roy, A.; Maiti, M.; Roy, S. Spontaneous Formation of Vesicles by Sodium 2Dodecylnicotinate in Water. Langmuir 2012, 28 (35), 12696-12703.

45. Wang, H.; Zhang, L.; Wang, J.; Li, Z.; Zhang, S. The first evidence for unilamellar vesicle formation of ionic liquids in aqueous solutions. Chem. Comm. 2013, 49 (45), 5222-5224.

46. Felgner, P. L.; Gadek, T. R.; Holm, M.; Roman, R.; Chan, H. W.; Wenz, M.; Northrop, J. P.; Ringold, G. M.; Danielsen, M. Lipofection: a highly efficient, lipid-mediated DNAtransfection procedure. PNAS 1987, 84 (21), 7413-7417.

47. Simões, S.; Filipe, A.; Faneca, H.; Mano, M.; Penacho, N.; Düzgünes, N.; Pedroso de Lima, M. Cationic liposomes for gene delivery. Expert Opin. Drug Delivery 2005, 2 (2), 237-254.

48. Kunstfeld, R.; Wickenhauser, G.; Michaelis, U.; Teifel, M.; Umek, W.; Naujoks, K.; Wolff, K.; Petzelbauer, P. Paclitaxel Encapsulated in Cationic Liposomes Diminishes Tumor Angiogenesis and Melanoma Growth in a "Humanized" SCID Mouse Model. Journal of Investigative Dermatology 2003, 120 (3), 476-482.

49. Stefanutti, E.; Papacci, F.; Sennato, S.; Bombelli, C.; Viola, I.; Bonincontro, A.; Bordi, F.; Mancini, G.; Gigli, G.; Risuleo, G. Cationic liposomes formulated with DMPC and a gemini surfactant traverse the cell membrane without causing a significant bio-damage. Biochim. Biophys. Acta, Biomembr. 2014, 1838 (10), 2646-2655.

50. Lichtenberg, D.; Ahyayauch, H.; Goñi, Félix M. The Mechanism of Detergent Solubilization of Lipid Bilayers. Biophys. J. 2013, 105 (2), 289-299.

51. Li, X.; Yang, Y.; Eastoe, J.; Dong, J. Rich Self-Assembly Behavior from a Simple Amphiphile. ChemPhysChem 2010, 11 (14), 3074-3077.

52. Yang, Y.; Dong, J.; Li, X. Micelle to vesicle transitions of N-dodecyl-1, $\omega$ diaminoalkanes: Effects of $\mathrm{pH}$, temperature and salt. J. Colloid Interface Sci. 2012, 380 (1), 83-89.

53. Goldsipe, A.; Blankschtein, D. Molecular-Thermodynamic Theory of Micellization of pH-Sensitive Surfactants. Langmuir 2006, 22 (8), 3547-3559.

54. Felber, A. E.; Dufresne, M.-H.; Leroux, J.-C. pH-sensitive vesicles, polymeric micelles, and nanospheres prepared with polycarboxylates. Adv. Drug Delivery Rev. 2012, 64 (11), 979-992.

55. He, X.; Li, J.; An, S.; Jiang, C. pH-sensitive drug-delivery systems for tumor targeting. Ther. Delivery 2013, 4 (12), 1499-1510.

56. de Duve, C.; Trouet, A.; Campeneere, D. D.; Baurian, R. Liposomes as lysosomotropic carriers. Ann N Y Acad Sci 1978, 308, 226-34. 
57. Yatvin, M. B.; Kreutz, W.; Horwitz, B. A.; Shinitzky, M. pH-sensitive liposomes: possible clinical implications. Science 1980, 210 (4475), 1253-5.

58. Connor, J.; Yatvin, M. B.; Huang, L. pH-sensitive liposomes: acid-induced liposome fusion. PNAS 1984, 81 (6), 1715-1718.

59. Schmaljohann, D. Thermo- and $\mathrm{pH}$-responsive polymers in drug delivery. Adv. Drug Delivery Rev. 2006, 58 (15), 1655-1670.

60. Nakayama, M.; Akimoto, J.; Okano, T. Polymeric micelles with stimuli-triggering systems for advanced cancer drug targeting. J. Drug Targeting 2014, 22 (7), 584-99.

61. Sampathkumar, K.; Arulkumar, S.; Ramalingam, M. Advances in Stimuli Responsive Nanobiomaterials for Cancer Therapy. J. Biomed. Nanotechnol. 2014, 10 (3), 367-382.

62. Discher, D. E.; Eisenberg, A. Polymer vesicles. Science 2002, 297 (5583), 967-73.

63. Peterca, M.; Percec, V.; Leowanawat, P.; Bertin, A. Predicting the Size and Properties of Dendrimersomes from the Lamellar Structure of Their Amphiphilic Janus Dendrimers. $J$. Am. Chem. Soc. 2011, 133 (50), 20507-20520.

64. Jhaveri, A.; Torchilin, V. Intracellular delivery of nanocarriers and targeting to subcellular organelles. Expert Opin. Drug Delivery 2016, 13 (1), 49-70.

65. Oh, N. M.; Oh, K. T.; Youn, Y. S.; Lee, D.-K.; Cha, K.-H.; Lee, D. H.; Lee, E. S. Poly(laspartic acid) nanogels for lysosome-selective antitumor drug delivery. Colloids Surf., $B$ 2013, 101, 298-306.

66. Giannotti, M. I.; Esteban, O.; Oliva, M.; García-Parajo, M. F.; Sanz, F. pH-Responsive Polysaccharide-Based Polyelectrolyte Complexes As Nanocarriers for Lysosomal Delivery of Therapeutic Proteins. Biomacromol. 2011, 12 (7), 2524-2533.

67. Zhu, S.; Lansakara-P, D. S. P.; Li, X.; Cui, Z. Lysosomal Delivery of a Lipophilic Gemcitabine Prodrug Using Novel Acid-Sensitive Micelles Improved Its Antitumor Activity. Bioconjugate Chem. 2012, 23 (5), 966-980.

68. Meerovich, I.; Koshkaryev, A.; Thekkedath, R.; Torchilin, V. P. Screening and Optimization of Ligand Conjugates for Lysosomal Targeting. Bioconjugate Chem. 2011, 22 (11), 2271-2282.

69. Wexselblatt, E.; Esko, J. D.; Tor, Y. GNeosomes: Highly Lysosomotropic Nanoassemblies for Lysosomal Delivery. ACS Nano 2015, 9 (4), 3961-3968.

70. Koshkaryev, A.; Thekkedath, R.; Pagano, C.; Meerovich, I.; Torchilin, V. P. Targeting of lysosomes by liposomes modified with octadecyl-rhodamine B. J. Drug Targeting 2011, 19 (8), 606-614.

71. Maniganda, S.; Sankar, V.; Nair, J. B.; Raghu, K. G.; Maiti, K. K. A lysosome-targeted drug delivery system based on sorbitol backbone towards efficient cancer therapy. Org. Biomol. Chem. 2014, 12 (34), 6564-6569.

72. Nair, J. B.; Mohapatra, S.; Ghosh, S.; Maiti, K. K. Novel lysosome targeted molecular transporter built on a guanidinium-poly-(propylene imine) hybrid dendron for efficient delivery of doxorubicin into cancer cells. Chem. Comm. 2015, 51 (12), 2403-2406. 
TOC graphic

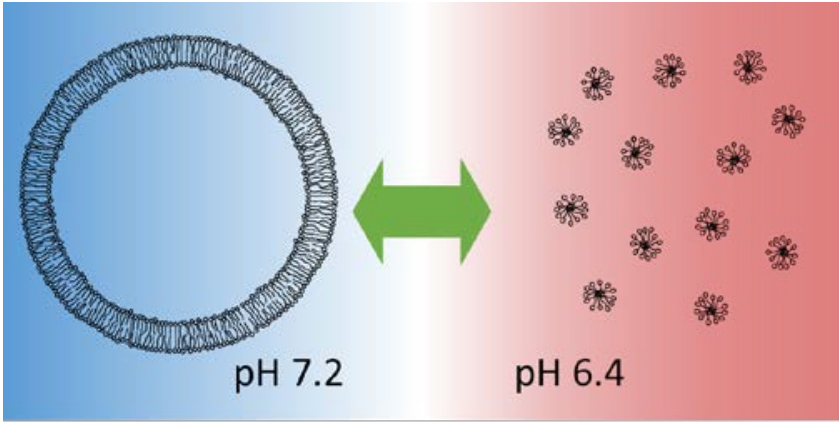

